\title{
A further discussion of the Hausdorff dimension in Engel expansions
}

\author{
by \\ Lu-Ming Shen (Wuhan and Changsha)
}

1. Introduction. In $\mathrm{GA}$, the Engel expansion transformation of $x \in$ $(0,1]$ was defined as $T x=d_{1}(x) x-1$, where $d_{1}(x)=[1 / x]+1$, and the partial quotients $\left\{d_{n}(x)\right\}_{n \geq 1}$ of the Engel expansion were defined by $d_{n}(x)=$ $d_{1}\left(T^{n-1}(x)\right)$. By the algorithm, one has $d_{j+1}(x) \geq d_{j}(x)$ for $j \geq 1$, and any $x \in(0,1]$ can be expanded as

$$
x=\frac{1}{d_{1}(x)}+\frac{1}{d_{1}(x) d_{2}(x)}+\cdots+\frac{1}{d_{1}(x) d_{2}(x) \cdots d_{n}(x)}+\cdots,
$$

which is denoted by $x=\left[d_{1}(x), d_{2}(x), \ldots\right]$ for short. In [GA], J. Galambos proved that for almost all $x \in(0,1]$,

$$
\lim _{n \rightarrow \infty} d_{n}(x)^{1 / n}=1 .
$$

Also, he posed the problem of finding the Hausdorff dimension of the set where (1.2) fails. In [WU], J. Wu proved that this Hausdorff dimension is 1. More generally, he proved that for any $\alpha \geq 1$, the Hausdorff dimension of the set

$$
A(\alpha)=\left\{x \in(0,1]: \lim _{n \rightarrow \infty} d(x)^{1 / n}=\alpha\right\}
$$

is 1. In this paper, we find the Hausdorff dimension of the set

$$
E(\alpha)=\left\{x \in(0,1]: \lim _{n \rightarrow \infty} \frac{\log d_{n+1}(x)}{\log d_{n}(x)}=\alpha\right\} .
$$

Theorem 1.1. For any $\alpha \geq 1, \operatorname{dim}_{\mathrm{H}} E(\alpha)=1 / \alpha$.

\section{The proof of the theorem}

2.1. Upper bound. Firstly, a simple but useful lemma is stated.

2010 Mathematics Subject Classification: Primary 11K55; Secondary 28A80.

Key words and phrases: Engel expansion, partial quotients, Hausdorff dimension. 
Lemma 2.1. Suppose $2 \leq d_{1} \leq d_{2} \leq \cdots \leq d_{n}$ are given integers, let $S \subset(0,1]$ and

$$
S^{\prime}=\left\{x^{\prime}: x^{\prime}=\frac{1}{d_{1}}+\frac{1}{d_{1} d_{2}}+\cdots+\frac{x}{d_{1} d_{2} \cdots d_{n}}, x \in S\right\} .
$$

Then $\operatorname{dim}_{\mathrm{H}} S=\operatorname{dim}_{\mathrm{H}} S^{\prime}$.

Proof. Define the map $S \rightarrow S^{\prime}$ by

$$
f_{d_{1} d_{2} \cdots d_{n}}(x)=\frac{1}{d_{1}}+\frac{1}{d_{1} d_{2}}+\cdots+\frac{x}{d_{1} d_{2} \cdots d_{n}} .
$$

Since

$$
\left|x_{1}^{\prime}-x_{2}^{\prime}\right|=\frac{\left|x_{1}-x_{2}\right|}{d_{1} d_{2} \cdots d_{n}} \quad \text { for any } x_{1}, x_{2} \in S,
$$

the map $f$ is bi-lipschitz, so $\operatorname{dim}_{\mathrm{H}} S=\operatorname{dim}_{\mathrm{H}} S^{\prime}$.

LEMma 2.2. $\operatorname{dim}_{\mathrm{H}} E(\alpha) \leq 1 / \alpha$.

Proof. By the definition of $E(\alpha)$, for any $x \in E(\alpha)$ and $\epsilon>0$, there exists $N \in \mathbb{N}$ such that $\left[d_{n}^{\alpha-\epsilon}(x)\right] \leq d_{n+1}(x) \leq\left[d_{n}^{\alpha+\epsilon}(x)\right]$ for any $n \geq N$. Take

$$
\begin{aligned}
E_{\epsilon}(\alpha) & =\bigcup_{N=1}^{\infty}\left\{x \in(0,1]:\left[d_{n}^{\alpha-\epsilon}(x)\right] \leq d_{n+1}(x) \leq\left[d_{n}^{\alpha+\epsilon}(x)\right], \forall n \geq N\right\} \\
& =\bigcup_{N=1}^{\infty} E_{\epsilon}(N, \epsilon) .
\end{aligned}
$$

Obviously, $E(\alpha) \subset E_{\epsilon}(\alpha)$ for any $0<\epsilon<\alpha$. By Lemma 2.1, $\operatorname{dim}_{\mathrm{H}} E_{\epsilon}(N, \alpha)$ $=\operatorname{dim}_{\mathrm{H}} E_{\epsilon}(1, \alpha)$ for any $N \geq 1$, thus

$$
\operatorname{dim}_{\mathrm{H}} E(\alpha) \leq \sup _{N \geq 1} \operatorname{dim}_{\mathrm{H}} E_{\epsilon}(N, \alpha)=\operatorname{dim}_{\mathrm{H}} E_{\epsilon}(1, \alpha) .
$$

In what follows, we use the symbolic space $D=\bigcup_{n=0}^{\infty} D_{n}$, where $D_{0}=\emptyset$ and for any $n \geq 1$,

$$
D_{n}=\left\{\left(\sigma_{1}, \ldots, \sigma_{n}\right) \in \mathbb{N}^{n}:\left[\sigma_{k}^{\alpha-\epsilon}\right] \leq \sigma_{k+1} \leq\left[\sigma_{k}^{\alpha+\epsilon}\right], \forall 1 \leq k \leq n-1\right\} .
$$

For any $\sigma=\left(\sigma_{1}, \ldots, \sigma_{n}\right) \in D_{n}$, we call the set

$$
I\left(\sigma_{1}, \ldots, \sigma_{n}\right):=\operatorname{cl}\left\{x \in(0,1]: d_{1}(x)=\sigma_{1}, \ldots, d_{n}(x)=\sigma_{n}\right\}
$$

an $n$th basic cylinder, and

$$
J_{\sigma}:=\bigcup_{\left[\sigma_{n}^{\alpha-\epsilon}\right] \leq \sigma_{n+1} \leq\left[\sigma_{n}^{\alpha+\epsilon}\right]} I\left(\sigma_{1}, \ldots, \sigma_{n}, \sigma_{n+1}\right)
$$


an $n$th basic interval. Then

$$
\begin{aligned}
\left|J_{\sigma}\right| & =\left|\frac{1}{\sigma_{1} \cdots \sigma_{n}\left[\sigma_{n}^{\alpha-\epsilon}\right]}-\frac{1}{\sigma_{1} \cdots \sigma_{n}\left[\sigma_{n}^{\alpha+\epsilon}\right]}\right| \\
& \leq \frac{1}{\sigma_{1} \cdots \sigma_{n-1}}\left(\frac{2}{\sigma_{n} \sigma_{n}^{\alpha-\epsilon}}-\frac{1}{\sigma_{n} \sigma_{n}^{\alpha+\epsilon}}\right) \leq \frac{4}{\sigma_{1} \cdots \sigma_{n-1} \sigma_{n}^{1+\alpha-\epsilon}} .
\end{aligned}
$$

Notice that

$$
E_{\epsilon}(1, \alpha)=\left\{x \in(0,1]:\left[\sigma_{n}^{\alpha-\epsilon}\right] \leq \sigma_{n+1} \leq\left[\sigma_{n}^{\alpha+\epsilon}\right], \forall n \geq 1\right\}=\bigcap_{n=1}^{\infty} \bigcup_{\sigma \in D_{n}} J_{\sigma} .
$$

Take $\epsilon>0$ so small that for any $n \geq 1$,

$$
\sum_{\left[\sigma_{n-1}^{\alpha-\epsilon}\right] \leq \sigma_{n} \leq\left[\sigma_{n-1}^{\alpha+\epsilon}\right]}\left(\frac{2}{\sigma_{n}^{\alpha-\epsilon}}\right)^{1 / \alpha} \leq 1
$$

Then

$$
\begin{aligned}
& \mathcal{H}^{1 / \alpha}\left(E_{\epsilon}(1, \alpha)\right) \leq \liminf _{n \rightarrow \infty} \sum_{\sigma \in D_{n}}\left|J_{\sigma}\right|^{1 / \alpha} \leq \liminf _{n \rightarrow \infty} \sum_{\sigma \in D_{n}}\left(\frac{4}{\sigma_{1} \cdots \sigma_{n-1} \sigma_{n}^{1+\alpha-\epsilon}}\right)^{1 / \alpha} \\
& =\liminf _{n \rightarrow \infty} \sum_{\left(\sigma_{1}, \ldots, \sigma_{n-1}\right) \in D_{n-1}}\left(\frac{4}{\sigma_{1} \cdots \sigma_{n-1}^{1+\alpha-\epsilon}}\right)^{1 / \alpha}\left(\frac{\sigma_{n-1}^{\alpha-\epsilon}}{\sigma_{n}^{1+\alpha-\epsilon}}\right)^{1 / \alpha} \\
& \leq \liminf _{n \rightarrow \infty} \sum_{\left(\sigma_{1}, \ldots, \sigma_{n-1}^{\alpha-\epsilon}\right) \in D_{n-1} \leq \sigma_{n} \leq\left[\sigma_{n-1}^{\alpha+\epsilon}\right]}\left(\frac{4}{\sigma_{1} \cdots \sigma_{n-1}^{1+\alpha-\epsilon}}\right)^{1 / \alpha} \sum_{\sum_{n \rightarrow \infty}}\left(\frac{2}{\sigma_{n}^{\alpha-\epsilon}}\right)^{1 / \alpha} \\
& \leq \liminf _{\left.\left(\sigma_{1}, \ldots, \sigma_{n-1}\right) \in D_{n-1}-\epsilon\right] \leq \sigma_{n} \leq\left[\sigma_{n-1}^{\alpha+\epsilon}\right]}\left(\frac{4}{\sigma_{1} \cdots \sigma_{n-2} \sigma_{n-1}^{1+\alpha-\epsilon}}\right)^{1 / \alpha}<\infty
\end{aligned}
$$

which implies $\operatorname{dim}_{\mathrm{H}} E(\alpha) \leq 1 / \alpha$.

2.2. Lower bound. We state the mass distribution principle first.

Lemma 2.3 (Distribution Principle, see also [FA, Proposition 2.3]). Let $E$ be a Borel set, and $\mu$ a measure with $\mu(E)>0$. If for any $x \in E$,

$$
\liminf _{r \rightarrow 0} \frac{\log \mu(B(x, r))}{\log r} \geq s,
$$

where $B(x, r)$ denotes the open ball with center at $x$ and radius $r$, then $\operatorname{dim}_{H} E \geq s$.

Recall that for $\alpha \geq 1$,

$$
E(\alpha)=\left\{x \in(0,1]: \lim _{n \rightarrow \infty} \frac{\log d_{n+1}(x)}{\log d_{n}(x)}=\alpha\right\}
$$


To give a lower bound of $\operatorname{dim}_{\mathrm{H}} E(\alpha)$, we define a subset of $E(\alpha)$ by

$$
F=\left\{x \in(0,1]: 2\left[d_{n}^{\alpha}(x)\right]+1 \leq d_{n+1}(x) \leq 3\left[d_{n}^{\alpha}(x)\right] \text { for all } n \geq 1\right\} .
$$

To exhibit the structure of the set $F$, we use the symbolic space $D=$ $\bigcup_{n=0}^{\infty} D_{n}$, where $D_{0}=\emptyset$ and for any $n \geq 1$,

$$
D_{n}=\left\{\left(\sigma_{1}, \ldots, \sigma_{n}\right) \in \mathbb{N}^{n}: 2\left[\sigma_{k}^{\alpha}\right]+1 \leq \sigma_{k+1} \leq 3\left[\sigma_{k}^{\alpha}\right], 1 \leq k<n\right\} .
$$

As in Section 2.1, for each $\sigma=\left(\sigma_{1}, \ldots, \sigma_{n}\right) \in D_{n}$, we call

$$
\begin{aligned}
& I\left(\sigma_{1}, \ldots, \sigma_{n}\right):=\operatorname{cl}\left\{x \in[0,1]: d_{1}(x)=\sigma_{1}, \ldots, d_{n}(x)=\sigma_{n}\right\}, \\
& J\left(\sigma_{1}, \ldots, \sigma_{n}\right):=\bigcup_{2\left[\sigma_{n}^{\alpha}\right]+1 \leq \sigma_{n+1} \leq 3\left[\sigma_{n}^{\alpha}\right]} I\left(\sigma_{1}, \ldots, \sigma_{n}, \sigma_{n+1}\right)
\end{aligned}
$$

an $n$th basic cylinder and $n$th basic interval respectively. Then by a simple computation, we have

$$
\left|I\left(\sigma_{1}, \ldots, \sigma_{n}\right)\right|=\frac{1}{\sigma_{1} \cdots \sigma_{n-1}\left(\sigma_{n}-1\right) \sigma_{n}},
$$

and

$$
\frac{1}{6} \frac{1}{\sigma_{1} \cdots \sigma_{n-1} \sigma_{n}^{\alpha+1}} \leq\left|J\left(\sigma_{1}, \ldots, \sigma_{n}\right)\right| \leq \frac{1}{3} \frac{1}{\sigma_{1} \cdots \sigma_{n-1} \sigma_{n}^{\alpha+1}},
$$

and one can observe that

$$
F=\bigcap_{n=1}^{\infty} \bigcup_{\left(\sigma_{1}, \ldots, \sigma_{n}\right) \in D_{n}} J\left(\sigma_{1}, \ldots, \sigma_{n}\right) .
$$

Lemma 2.4. $\operatorname{dim}_{\mathrm{H}} E(\alpha) \geq 1 / \alpha$.

Proof. For each $n \geq 1$ and $\sigma=\left(\sigma_{1}, \ldots, \sigma_{n}\right) \in D_{n}$, denote by $g^{\ell}\left(\sigma_{1}, \ldots, \sigma_{n}\right)$ the length of the gap between the left endpoint of $I\left(\sigma_{1}, \ldots, \sigma_{n}\right)$ and the left endpoint of $J\left(\sigma_{1}, \ldots, \sigma_{n}\right)$ and by $g^{r}\left(\sigma_{1}, \ldots, \sigma_{n}\right)$ the length of the gap between the right endpoint of $I\left(\sigma_{1}, \ldots, \sigma_{n}\right)$ and the right endpoint of $J\left(\sigma_{1}, \ldots, \sigma_{n}\right)$. Finally, let

$$
G\left(\sigma_{1}, \ldots, \sigma_{n}\right):=\min \left\{g^{\ell}\left(\sigma_{1}, \ldots, \sigma_{n}\right), g^{r}\left(\sigma_{1}, \ldots, \sigma_{n}\right)\right\} .
$$

Then

$$
\frac{1}{3} \frac{1}{\sigma_{1} \cdots \sigma_{n-1} \sigma_{n}^{\alpha+1}} \leq G\left(\sigma_{1}, \ldots, \sigma_{n}\right) \leq \frac{2}{3} \frac{1}{\sigma_{1} \cdots \sigma_{n-1} \sigma_{n}^{\alpha+1}} .
$$

Now, we define a probability measure on $F$. The set function $\mu:\{J(\sigma)$ : $\left.\sigma \in D \backslash D_{0}\right\} \rightarrow \mathbb{R}^{+}$is given by

$$
\mu\left(J\left(\sigma_{1}, \ldots, \sigma_{n}\right)\right)=\frac{1}{\left[\sigma_{n-1}^{\alpha}\right]} \mu\left(J\left(\sigma_{1}, \ldots, \sigma_{n-1}\right)\right)=\prod_{k=1}^{n-1} \frac{1}{\left[\sigma_{k}^{\alpha}\right]},
$$

and

$$
\mu\left(J\left(\sigma_{1}\right)\right)=\frac{1}{\sharp D_{1}},
$$


where $\sharp$ denotes cardinality. It can be easily verified that

$$
\mu\left(J\left(\sigma_{1}, \ldots, \sigma_{n-1}\right)\right)=\sum_{\sigma_{n}} \mu\left(J\left(\sigma_{1}, \ldots, \sigma_{n}\right)\right),
$$

where the summation is taken over all $\sigma_{n}$ such that $\left(\sigma_{1}, \ldots, \sigma_{n-1}, \sigma_{n}\right) \in D_{n}$. Notice that $\sum_{\sigma_{1} \in D_{1}} \mu\left(J\left(\sigma_{1}\right)\right)=1$, so, by Kolmogorov's extension theorem, the function $\mu$ can be extended to a probability measure supported on $F$, which is still denoted by $\mu$.

For each $x \in F$, there exists a sequence $\left(\sigma_{n}\right)_{n \geq 1}$ such that $\left(\sigma_{1}, \ldots, \sigma_{n}\right) \in$ $D_{n}$ and $x \in J\left(\sigma_{1}, \ldots, \sigma_{n}\right)$, for each $n \geq 1$. Assume that $r>0$ is small enough and let $n$ be the integer such that

$$
G\left(\sigma_{1}, \ldots, \sigma_{n+1}\right) \leq r<G\left(\sigma_{1}, \ldots, \sigma_{n}\right) .
$$

By the definition of $G$, it follows that the ball $B(x, r)$ can only intersect one $n$th basic cylinder $I\left(\sigma_{1}, \ldots, \sigma_{n}\right)$.

The following relationship can be verified easily. For any $\epsilon>0$, there exists $n \geq 1$ such that for each $\left(\sigma_{1}, \ldots, \sigma_{n}\right) \in D_{n}$,

$$
\prod_{k=1}^{n-1} \frac{1}{\left[\sigma_{k}^{\alpha}\right]} \leq\left(\frac{1}{\sigma_{1} \cdots \sigma_{n-1} \sigma_{n}^{\alpha+1}}\right)^{1 /(\alpha+\epsilon)} .
$$

Two cases will be distinguished.

(i) $G\left(\sigma_{1}, \ldots, \sigma_{n+1}\right) \leq r<\left|I\left(\sigma_{1}, \ldots, \sigma_{n+1}\right)\right|$. In this case, the ball $B(x, r)$ can intersect at most eight $(n+1)$ th basic cylinders contained in $I\left(\sigma_{1}, \ldots, \sigma_{n}\right)$. So,

$$
\mu(B(x, r)) \leq 8 \mu\left(J\left(\sigma_{1}, \ldots, \sigma_{n+1}\right)\right)=8 \prod_{k=1}^{n} \frac{1}{\left[\sigma_{k}^{\alpha}\right]} \leq 24 r^{1 /(\alpha+\epsilon)} .
$$

(ii) $\left|I\left(\sigma_{1}, \ldots, \sigma_{n+1}\right)\right| \leq r<G\left(\sigma_{1}, \ldots, \sigma_{n}\right)$. Notice that

$$
\min \left\{\left|I\left(\sigma_{1}, \ldots, \sigma_{n}, \bar{\sigma}_{n+1}\right)\right|: 2\left[\sigma_{n}^{\alpha}\right]+1 \leq \bar{\sigma}_{n+1} \leq 3\left[\sigma_{n}^{\alpha}\right]\right\} \geq \frac{1}{9 \sigma_{1} \cdots \sigma_{n-1} \sigma_{n}^{2 \alpha+1}} .
$$

In this case, $B(x, r)$ can intersect at most

$$
18 r \sigma_{1} \cdots \sigma_{n-1} \sigma_{n}^{2 \alpha+1}+2 \leq 54 r \sigma_{1} \cdots \sigma_{n-1} \sigma_{n}^{2 \alpha+1} \leq\left[72 r \sigma_{1} \cdots \sigma_{n-1} \sigma_{n}^{2 \alpha+1}\right]
$$

$(n+1)$ th basic cylinders contained in $I\left(\sigma_{1}, \ldots, \sigma_{n}\right)$. So,

$$
\begin{aligned}
\mu & (B(x, r)) \\
& \leq \min \left\{\mu\left(J\left(\sigma_{1}, \ldots, \sigma_{n}\right)\right),\left[72 r \sigma_{1} \cdots \sigma_{n-1} \sigma_{n}^{2 \alpha+1}\right] \mu\left(J\left(\sigma_{1}, \ldots, \sigma_{n+1}\right)\right)\right\} \\
& \leq \prod_{k=1}^{n-1} \frac{1}{\left[\sigma_{k}^{\alpha}\right]} \min \left\{1,72 r \sigma_{1} \cdots \sigma_{n-1} \sigma_{n}^{2 \alpha+1} \frac{1}{\left[\sigma_{n}^{\alpha}\right]}\right\}
\end{aligned}
$$




$$
\begin{aligned}
& \leq \prod_{k=1}^{n-1} \frac{1}{\left[\sigma_{k}^{\alpha}\right]} \min \left\{1,114 r \sigma_{1} \cdots \sigma_{n-1} \sigma_{n}^{\alpha+1}\right\} \quad\left(\text { as } \min \{a, b\} \leq a^{s} b^{1-s}, 0<s<1\right) \\
& \leq \prod_{k=1}^{n-1} \frac{1}{\left[\sigma_{k}^{\alpha}\right]}\left(114 r \sigma_{1} \cdots \sigma_{n-1} \sigma_{n}^{\alpha+1}\right)^{1 /(\alpha+\epsilon)} \quad(\text { by }(2.1)) \\
& \leq 114^{1 /(\alpha+\epsilon)} r^{1 /(\alpha+\epsilon)} .
\end{aligned}
$$

By Lemma $2.3, \operatorname{dim}_{H} F \geq 1 /(\alpha+\epsilon)$. Since $\epsilon$ is arbitrary, $\operatorname{dim}_{H} E(\alpha) \geq$ $\operatorname{dim}_{\mathrm{H}} F \geq 1 / \alpha$. Combining this with Lemmas 2.2 and 2.4, we obtain Theorem 1.1 .

Acknowledgements. This research was partly supported by NSFC (grant no. 10631040), the Hunan Nature Science (grant no. 09JJ3001) and the Technical Plan Project of Hunan Province (grant no. 2008FJ3046).

We also thank Dr. Baowei Wang and the referee for helpful suggestions.

\section{References}

[FA] K. J. Falconer, Techniques in Fractal Geometry, Wiley, New York, 1997.

[GA] J. Galambos, Representations of Real Numbers by Infinite Series, Lecture Notes in Math. 502, Springer, Berlin, 1976.

[WU] J. Wu, A problem of Galambos on Engel expansions, Acta Arith. 92 (2000), 383386.

Lu-ming Shen

School of Mathematics and Statistics

Huazhong University of Science and Technology

Wuhan, Hubei, 430072, P.R. China

and

Science College of Hunan Agriculture University

Changsha, Hunan, 410128, P.R. China

E-mail: lum_s@126.com 